\title{
Genesis
}

Manuscrits - Recherche - Invention

\section{Almuth Grésillon, Marie-Madeleine Mervant-Roux, Dominique Budor (dir.), Genèses théâtrales, Paris, CNRS Éditions, coll. « Textes et Manuscrits », 2010, $270 \mathrm{p}$.}

Florent Siaud

\section{(2) OpenEdition}

\section{Journals}

Édition électronique

URL : http://journals.openedition.org/genesis/639

DOI : 10.4000/genesis.639

ISSN : 2268-1590

Éditeur :

Presses universitaires de Paris Sorbonne (PUPS), Société internationale de génétique artistique littéraire et scientifique (SIGALES)

\section{Édition imprimée}

Date de publication : 30 octobre 2011

Pagination : 187-189

ISBN : 978-2-84050-804-5

ISSN : 1167-5101

\section{Référence électronique}

Florent Siaud, «Almuth Grésillon, Marie-Madeleine Mervant-Roux, Dominique Budor (dir.), Genèses théâtrales, Paris, CNRS Éditions, coll. «Textes et Manuscrits », 2010, 270 p. », Genesis [En ligne], 33 2011, mis en ligne le 23 octobre 2013, consulté le 22 septembre 2020. URL : http:// journals.openedition.org/genesis/639; DOI : https://doi.org/10.4000/genesis.639 


\section{Comptes rendus d'ouvrages}

\begin{abstract}
Almuth Grésillon, Marie-Madeleine Mervant-Roux, Dominique Budor (dir.), Genèses théâtrales, Paris, CNRS Éditions, coll. « Textes et Manuscrits », 2010, 270 p.

\section{Compte rendu par Florent Siaud}

Alors que la génétique textuelle analyse des avant-textes d'écrivains depuis les années soixante-dix, l'attention portée à la genèse théâtrale à proprement parler est plus récente. Plusieurs paramètres sont susceptibles d'expliquer ce décalage temporel. Le plus important tient peutêtre à la polymorphie caractéristique de l'œuvre théâtrale : approcher sa gestation ne consiste plus seulement à restituer les mouvements d'une écriture qui se cherche dans un atelier solitaire - c'était et cela demeure l'objectif, déjà considérable, de la génétique appliquée à des corpus d'archives exclusivement textuelles. L'opération implique que l'on prenne en compte la pluralité des subjectivités engagées dans la création théâtrale, les modifications apportées au texte par la mise en scène ou encore la multiplicité des supports disponibles (brouillons de dramaturge certes, mais aussi carnets de notes, sténogrammes de répétitions, images vidéographiques, captations sonores, photographies, croquis et autres esquisses). Autant de défis posés à la méthodologie des chercheurs, parfois désarçonnés par le caractère insaisissable d'un tel objet d'étude. Loin d'éluder ces difficultés, les trois directrices de publications de Genèses théâtrales ont choisi de les affronter en optant pour un titre au pluriel qui sonne d'emblée comme un manifeste : on ne saurait parler d'une genèse théâtrale ; il y en a nécessairement plusieurs, puisque à une approche textuelle de la création théâtrale se surimprime nécessairement ce qu'il est désormais convenu d'appeler une " génétique du spectacle ».

Récusant les binarismes réducteurs (texte/scène, auteur dramatique/metteur en scène), Almuth Grésillon, MarieMadeleine Mervant-Roux et Dominique Budor délimitent le cadre de cet ouvrage collectif en plaidant pour une approche interdisciplinaire, qui n'occulte ni les différentes « constellations de recherches » ni la polyphonie à travers laquelle tout projet théâtral prend corps. $\mathrm{Au}$ modèle de l'œuvre achevée, elles entendent substituer l'image dynamique d'une entité en mouvement permanent. Cette révolution copernicienne passe par la prise en compte de corpus d'archives aux formats variés mais aussi de témoignages de créateurs.

C'est à ces derniers que la première partie du recueil est justement consacrée. Brigitte Jaques-Wajeman y prend la parole pour y décrire la gestation d'une mise en scène de Britannicus au Théâtre du Vieux-Colombier. Plongeant le lecteur dans l'intimité de ses préparatifs et lui ouvrant les pages d'un carnet de notes personnelles, elle évoque ses lectures, avant de se remémorer les premiers ateliers de travail avec les acteurs, sa collaboration avec le costumier et décorateur Emmanuel Peduzzi, ainsi que les changements de distribution à la reprise du spectacle.

$\mathrm{Au}$ témoignage de Jean Jourdheuil, retraçant l'histoire d'une commande passée par le Festival d'automne autour de l'œuvre non dramatique de Michel Foucault succède celui de Jacques Lassalle. L'ancien administrateur de la Comédie-
Française y évoque avec poésie et pudeur les tiraillements fascinants de sa direction d'acteur : tendue entre le « trop d'amour» et le « trop d'inassouvi », marquée par les paradoxes, elle est ouverte à l'errance, la rature et l'abandon. Regrettant que le compagnonnage entre praticiens et exégètes soit moins fort que par le passé, il pose la question de la mémoire du théâtre et du témoignage sur les répétitions : le film documentaire ou le suivi intégral des répétitions par un observateur actif lui semblent les deux voies envisageables.

À la croisée du monde du spectacle et l'univers de la recherche, Sophie Proust se propose d'établir une typologie des écrits de l'assistant à la mise en scène à partir d'un savoir empiriquement constitué auprès de metteurs en scène comme Yves Beaunesne, Denis Marleau, Matthias Langhoff et Robert Wilson. Elle les range dans trois grandes catégories : les écrits produits en amont, pendant et après les répétitions. Le détour par la taxinomie lui permet de rationaliser une multiplicité de vestiges écrits, de nature artistique, technique, logistique ou administrative. On regrette seulement que la passionnante référence à la démarche ethnographique de Gay McAuley ne soit pas exploitée davantage.

Ancienne directrice du département des Arts du spectacle de la Bibliothèque nationale de France, Noëlle Guibert ouvre la partie consacrée aux «paroles de chercheurs ». Elle voit dans les manuscrits de copistes, les correspondances, les maquettes de costumes et de décors autant de traces de processus créateurs qui, aux $\mathrm{XVIII}^{\mathrm{e}}$ et XIXe siècles, sont coordonnés par des figures d'acteurs (Lekain, Talma) et 
d'auteurs dramatiques (Vigny, Dumas, Hugo), avant d'être dirigés par les metteurs en scène dès la fin du XIXe siècle.

À cette mise en perspective des ressources archivistiques succède une analyse des documents de Luigi Pirandello, signée Dominique Budor. Composés de publications ignorées, de lettres, de contrats et de documents autographes, ceux-ci ne donnent pas seulement à entendre la voix de leur auteur mais aussi celle de son collaborateur et fils Stefano. La notion d'auctorialité s'y trouve redéfinie. Remettant en cause l'idée d'une « intentionnalité globale de l'œuvre » comme celle d'un « fil rouge », ces écrits à quatre mains laissent sourdre un dialogisme et une indétermination qui inflige un coup sévère à une vision monologique et téléologique du processus d'écriture.

Après Pirandello, l'attention se déplace vers les œuvres de Jean Anouilh. Bernard Beugnot propose d'en appréhender la genèse en analysant des carnets, des pages manuscrites, des tapuscrits et des exemplaires annotés par l'écrivain. Loin de s'en tenir à ces documents, il porte également son attention sur la réécriture des didascalies, les témoignages d'artistes et les cahiers de mise en scène. Une intention, d'ailleurs récurrente à l'échelle de tout le recueil, s'y devine : voir comment le texte se métamorphose sous l'action des répétitions.

Dirk Van Hulle se demande pour sa part dans quelle mesure l'avant-texte qui préoccupe les généticiens ne correspondrait pas à ce que le romancier Ian McEwan appelle « l'obscène », soit ce qui se passe dans les coulisses. Pour conforter son hypothèse, il convoque les réflexions iconoclastes de Michel Foucault sur la définition de l'auteur. « Figure idéologique par laquelle on conjure la prolifération du sens », sa fonction peut être redéfinie à l'aune des travaux de la génétique : et si la génétique était une façon de restituer cette prolifération du sens ? Audacieuse et défendue avec aplomb, cette problématique est ici explorée via un corpus composé de textes de Beckett.
Quatrième auteur dramatique à être approché, Vinaver se distingue par sa volonté de publier les différentes versions d'une même pièce. Par-dessus bord en est le cas le plus symptomatique. De la collecte de documents à la publication des différentes versions, en passant par les phases de l'écriture et les mises en scène, Simon Chemama montre que les versions d'une même œuvre ne s'annulent pas les unes les autres mais qu'elles coexistent presque indépendamment. C'est la permanence et l'unicité de l'œuvre finale qui se voient ici relativisées.

Dévolue à la collaboration de Tchekhov et Stanislavski, l'étude de Marie-Christine Autant-Mathieu envisage de son côté l'émergence de la mise en scène comme un lent processus de maturation. Décrivant la façon dont le père de la méthode des actions physiques rédigeait ses mises en scène et se posait moins en dictateur qu'en pédagogue, elle nous plonge dans les préparatifs de La Mouette au Théâtre d'Art.

La présence de la génétique appliquée à un corpus russe est salutairement prolongée par Olga Anokhina, qui se fonde sur le Molière de Boulgakov pour explorer le concept d'《 exogenèse ». Récusant tout repli de l'acte d'écriture sur lui-même, elle démontre avec énergie comment l'idéologie, la censure et le travail de mise en scène peuvent avoir une incidence déterminante sur l'écriture d'une pièce de théâtre.

Almuth Grésillon et Marie-Madeleine Mervant-Roux se consacrent quant à elles au « torchon magnifique » de L'Amante anglaise. Imaginé par Marguerite Duras pour désigner l'exemplaire abondamment raturé et annoté de son œuvre par Michael Lonsdale, cet appellatif fait référence à un document portant trace des discussions des multiples intervenants du projet. Ayant presque le statut d'un objet d'art à part entière, celui-ci témoigne à lui seul des rapports dialectiques qui se sont instaurés entre genèse textuelle et genèse scénique pendant les répétitions de ce spectacle créé par Claude Régy.
La conclusion du recueil revient à Jean-Marie Thomasseau, qui propose d'introduire une nouvelle distinction dans le champ des études génétiques : un clivage entre théâtre contemporain et théâtre non contemporain. Loin de n'être qu'un constat technique, ce distinguo novateur invite à repenser le regard que l'histoire pose traditionnellement sur le théâtre passé : écho d'une auctorialité diffractée et porteur d'un mouvement de translation de la page vers la scène, l'histoire du théâtre ne doit plus se réduire à une histoire des textes, ni à une succession d'auteurs. L'histoire d'un mouvement, plutôt qu'une histoire des formes - celle-là même que pratiquent traditionnellement les études littéraires : tel devrait être son dessein.

Enrichi d'un glossaire et d'une bibliographie sélective éclairant les enjeux du recueil, Genèses théâtrales déploie une série de planches qui, par un habile jeu de renvoi, étayent le propos de chacun des contributeurs : des maquettes de décor du Britannicus de Brigitte Jaques-Wajeman aux notes d'assistante à la mise en scène de Sophie Proust, en passant par les couleurs chamarrées du «torchon magnifique » de L'Amante anglaise, les images convoquées démontrent que la génétique théâtrale doit désormais compter avec une diversité de supports qui sont loin d'avoir l'apparente et relative homogénéité des archives textuelles. À cet égard, l'hétérogénéité des corpus théâtraux semble encore mieux acceptée que dans le numéro 26 de la revue Genesis (2005), tournant déjà essentiel dans l'exploration de la genèse au théâtre. Toutefois, une question demeure peutêtre en suspens : au-delà de l'étude des photographies, des avant-textes de pièces de théâtre, des brochures annotées par l'équipe de mise en scène ou des plannings de répétitions réalisés par l'assistant, quelle place accorder au fugitif, à l'immatérialité et à l'expérience humaine qui se logent pourtant au cœur du processus créatif de tout spectacle vivant ? Empruntée à un congrès de la SIBMAS et convoquée dans l'introduction, la passionnante notion de « patrimoine immatériel » demanderait un 
développement supplémentaire. Genèses théâtrales constitue en somme un jalon majeur dans une enquête sur la création théâtrale que, loin de clore, il relance avec une ambition et une force inédites.

Hubert Thüring, Corinna Jäger-Trees, Michael Schläfli (dir.), Anfangen zu schreiben. Ein kardinales Moment von Textgenese und Schreibprozess im literarischen Archiv des 20. Jahrhunderts [Commencer à écrire/Une étape essentielle dans la genèse du texte et le processus d'écriture, d'après les archives littéraires du $\mathbf{x x}^{\mathrm{e}}$ siècle], München, Fink Verlag, 2009, 345 p.

\section{Compte rendu par Louis Hay}

On ne sait pas toujours en France que la Suisse aussi est terre de génétique. En Romandie, les chercheurs travaillent de longue date sur les archives littéraires fondées à Lausanne autour du fonds Ramuz. À Berne, le «Schweizerisches Literaturarchiv », créé à l'initiative de Dürrenmatt, a lancé un programme intitulé « Textgenese und Schreibprozess » auquel revient l'initiative du colloque de 2007 dont rend compte le présent volume de la collection "Zur Genealogie des Schreibens ». La rencontre porte sur une problématique classique de la critique génétique, celle des lieux stratégiques de l'écriture, un sujet qui a d'ailleurs été discuté dans plusieurs séminaires de l'ITEM 1 . C'est le problème des débuts qui est à l'ordre du jour ici et qui induit une grande variété d'approches.

Plusieurs études visent une typologie des débuts, qui s'étend du programmatique au processuel. Particulièrement intéressante est à cet égard la contribution de Corinna Jäger-Trees consacrée aux manuscrits du romancier suisse Otto F. Walter : l'écrivain y passe d'une écriture programmée (Der Stumme, 1959) à une rédaction immédiate (Zeit des Fasans, 1988). Notre habitude d'attribuer une écriture caractéristique à tout écrivain mérite peut-être réflexion.
Bernhild Boie analyse trois « entrées en écriture » parfaitement dissemblables (Heym, Eich, Rühmkorf). Écartant toute ambition typologique elle conclut, à l'instar de Gracq : «il n’y a que des cas d'espèce ». Cette position se trouve nuancée ailleurs : Hubert Thüring affirme que tout écrivain pense à la réception de son œuvre lorsqu'i commence à écrire, Stephan Kammer voit dans l'écriture une " technique culturelle complexe » et non une simple entreprise individuelle.

D'autres essais portent sur les fonctions esthétiques des commencements. Dans un manuscrit de Paul Celan, Sandro Zanetti souligne la fonction du titre, apparu à la fin de la genèse mais placé au début du texte pour en rappeler le statut initial : un « Projet», une « Ébauche ». Chez Robert Walser, Wolfram Groddeck trouve dans le texte même une contrainte qui s'impose au début : l'impossibilité d'annoncer une construction logique pour le livre à venir. Et dans les Notices d'Elias, Irmgard Wirtz retrouve les raisons qui rendent impossible l'existence même d'un début «Ce qui doit ici recevoir une durée, c'est le "Texte-Vie" qui ne doit comporter nul début, car la fin alors deviendrait visible », écrit l'auteur.

En changeant de thématique, un dernier groupe de contributions s'intéresse au commencement de l'écriture en tant que sujet littéraire. Hubert Thüring observe, dans les romans de Glauser, une « spirale » qui relie le mouvement de l'écriture à des éléments d'autoreprésentation. Dans les débuts de Kafka, Alexander Honold relève une réflexion sur l'acte d'écrire qui accompagne constamment le mouvement de la plume. Andreas Maunz enfin, s'intéresse aux écrits de Vassula Ryden, une mystique de l'Église grecque orthodoxe. Dans son recueil de messages True Life in God (1986-2003) il n'est, pour l'écrit, ni de début ni de fin puisque tout message de Dieu manifeste sa propre complétude. La tentative d'une " poétologie de l'écriture sacrée » se trouve cependant bornée par l'horizon du texte ; les manuscrits demeurent hors de portée.
Dans leur ensemble, les contributions du volume sont d'excellente qualité, même si les plus intéressantes se trouvent parmi les études de corpus les plus poussées. Le livre se recommande à tous les généticiens germanophones : il montre parfaitement tout ce qui reste à faire sur l'un des sujets les mieux étudiés de la génétique.

Brian Stimpson, Paul Valéry. L'écriture en devenir, Frankfurt am Main, Peter Lang, 2009, 432 p.

\section{Compte rendu par Jean-Michel} Maulpoix

Davantage que ses œuvres, si accomplies soient-elles, il se pourrait que ce soit le travail d'un écrivain qui le garde vivant, longtemps après sa disparition. Ainsi ne donne-t-il pas seulement à lire des textes, mais à interroger encore un geste de plume qui paraît se poursuivre sous nos yeux. Il en va ainsi pour Paul Valéry, tel que Brian Stimpson nous invite à le redécouvrir en rouvrant dans un volumineux essai intitulé L'Écriture en devenir le chantier de La Jeune Parque et de « La Pythie », à la lumière de documents manuscrits inédits parmi lesquels des lettres que Valéry a adressées à sa femme entre 1908 et 1918.

Brian Stimpson a suivi d'une façon très attentive (l'on est tenté d'écrire « très attentionnée ») Paul Valéry au travail, en cheminant à travers les brouillons de ses poèmes et sa correspondance, en jetant un double regard sur la poussée génétique manifeste dans les manuscrits qui permet d'appréhender l'évolution de l'écriture, et sur le processus de composition qui conduit à interroger plus globalement les moyens et la pratique de l'écrivain. Ainsi éclaire-t-il non seulement les textes, mais également la

1. Voir notamment Bernhild Boie et Daniel Ferrer (dir.), Genèses du roman contemporain. Incipit et entrée en écriture, Paris, CNRS Éditions, 2e éd., 1993 ; Claude Duchet et Isabelle Tournier (dir.), Genèses des fins, SaintDenis, PUV, 1996. 